\title{
A self-consistent analytical model for non-collisional heating
}

\author{
Shahid Rauf $\dagger$ and Mark J Kushner $\ddagger$ \\ University of Illinois, Department of Electrical and Computer Engineering, \\ 1406 W Green Street, Urbana, IL 61801, USA
}

Received 6 January 1997, in final form 3 July 1997

\begin{abstract}
Non-collisional heating is an important phenomenon in low-pressure inductively coupled plasmas. In this paper, a self-consistent analytical model for non-collisional heating is developed which shows that non-collisional heating is a warm-electron effect that relies on a resonant interaction of electrons with the electromagnetic wave. Electrons with velocities comparable to the effective wave phase speed resonantly interact with the wave and gain net energy from it. Collisions randomize this directed energy thereby leading to electron heating. Non-collisional heating becomes more efficient if a larger proportion of electrons participate in the resonant interaction process. This can occur due to an increase in the electron temperature or decrease in the effective wave phase speed. Collisions destroy the essential phase correlation between the electrons and the wave, making the non-collisional heating process less efficient.
\end{abstract}

\section{Introduction}

Non-collisional heating is an important electromagnetic phenomenon in low-pressure inductively coupled plasmas (ICPs) as used in industrial applications such as plasma materials processing [1-6]. It has been proposed that non-collisional heating occurs when warm electrons gain net energy while they traverse regions of inhomogeneous electric fields. If collisions are sparse and the electron mean free path is longer than the scale length of electric field absorption, this directed energy is randomized (i.e. electrons are heated) non-locally in the bulk of the plasma. In this paper, we further investigate the mechanism of noncollisional heating and the associated electron dynamics by means of a self-consistent kinetic model.

Non-collisional heating in the context of lowtemperature plasmas has received significant new attention in the past few years. Non-collisional heating has been explored in considerable detail in the past as an anamolous skin effect [7] as described by Weibel [8]. Using a kinetic model, he demonstrated that classical expressions for skin depth and surface impedance fail in a warm collisionless plasma. He then kinetically explored the electric field in the skin layer of a warm plasma and recomputed the surface impedance. The present interest in non-collisional (or hybrid) heating is a consequence of the wide adoption of ICP sources for etching and deposition by the microelectronics industry. Turner [1] was the first to popularize that non-collisional heating mechanisms might compete (or even dominate) collisional heating in the

\footnotetext{
† E-mail address: rauf@uigela.ece.uiuc.edu

† E-mail address: mjk@uiuc.edu
}

parameter regime (pressure and characteristic dimensions) where ICP sources are generally operated. He attributed this behaviour to warm-plasma effects. Godyak et al [2] observed evidence of non-collisional heating during electrical measurements in ICP reactors. Shaing [5] has recently used a kinetic model to derive a general expression for surface impedance that is valid in both collisional and non-collisional regimes. Non-collisional heating effects were first included in a large-scale ICP simulation by Vahedi et al [3]. Their approach was to define an effective collisional frequency which would take into account noncollisional heating. Rauf and Kushner [4] included noncollisional heating effects in an ICP simulation by means of the electron current density. This current density, which was computed kinetically using a Monte Carlo simulation, included contribution from all electron heating mechanisms. It was used as a source for the computation of the electric fields. Kolobov et al [6] have recently investigated noncollisional heating using a non-local model.

In this paper, we use a self-consistent kinetic model to look at the electron dynamics in the skin layer of a warm plasma. We find that non-collisional heating can be described as a warm-electron effect that relies on a resonant interaction of electrons with the electromagnetic wave. Electrons with velocities comparable to the 'effective' wave phase speed resonantly interact with the wave and gain net energy from it. Collisions randomize this directed energy, leading to electron heating. We also explore the effect of important plasma and wave characteristics on non-collisional heating. It is shown that non-collisional heating becomes more efficient as the proportion of electrons participating in the resonant interaction process increases. This can occur due to an increase in electron 
temperature or a reduction in the effective wave phase speed (resulting from a decrease in frequency or an increase in electron density). A phase correlation between the wave and electrons is essential for the resonant interaction process. Collisions destroy this phase correlation and reduce the efficiency of non-collisional heating. The analytical model also yields an expression for warmplasma conductivity which accounts for non-collisional heating. We demonstrate the utility of this expression by incorporating it into a general plasma equipment model.

In section 2, we describe the model and solve the equations. In section 3 , we use this model to understand the mechanism of non-collisional heating, and the influence of plasma and wave parameters on it. In section 4, we apply the model of section 2 to a plasma equipment simulation. Section 5 contains a brief summary.

\section{Description of the model}

In this section, we describe the plasma model and solve the resulting equations. This analysis will be connected to noncollisional heating in section 3. Consider a one-dimensional (1D) scenario in which there is a plasma-vacuum interface at the $z=0$ plane. The positive $z$ direction points towards the plasma. A $z$-directed $(\boldsymbol{k}=k \boldsymbol{z}) y$-polarized $\left(\boldsymbol{E}=E_{y} \boldsymbol{y}\right)$ plane electromagnetic wave is assumed to impinge on the interface from the vacuum side and penetrate into the plasma. To compute the fields within the plasma, our model consists of Maxwell's equations and Boltzmann's equation for electrons,

$$
\frac{\partial f_{e}}{\partial t}+(\boldsymbol{v} \cdot \nabla) f_{e}+\frac{q_{e}}{m_{e}}(\boldsymbol{E}+\boldsymbol{v} \times \boldsymbol{B}) \cdot \nabla_{\boldsymbol{v}} f_{e}=-v f_{e}
$$

where $f_{e}(\boldsymbol{r}, \boldsymbol{v}, t), v, q_{e}, \boldsymbol{E}$ and $\boldsymbol{B}$ are, respectively, the electron distribution function, electron momentum transfer collision frequency, electron charge, electric field and magnetic field. To simplify the model, we assume quasineutrality and neglect the displacement current. Maxwell's equations and (1) are coupled through the current density $\boldsymbol{J}$

$$
\boldsymbol{J}=q_{e} n_{e} \int \boldsymbol{v} f_{e} \mathrm{~d} v
$$

where $n_{e}$ is the electron density. We neglect the ion current due to the low ion mobility. The collision frequency and electron density are assumed to be constant throughout the plasma.

Linearizing (1), assuming that all quantities vary as $\exp (-\mathrm{i} \omega t)$, taking the Fourier transform in space and making use of Maxwell's equations, we obtain

$$
\boldsymbol{J}=\underline{\sigma} \cdot \boldsymbol{E}
$$

where

$$
\begin{aligned}
\boldsymbol{\sigma}= & \frac{\mathrm{i} q_{e}^{2} n_{e}}{m_{e}} \int \mathrm{d} \boldsymbol{v} \frac{\boldsymbol{v}}{\boldsymbol{k} \cdot \boldsymbol{v}-\omega-\mathrm{i} v} \nabla_{\boldsymbol{v}} f_{0} \\
& \cdot\left[\left(1-\frac{\boldsymbol{k} \cdot \boldsymbol{v}}{\omega}\right) \underline{I}+\frac{\boldsymbol{k} \boldsymbol{v}}{\omega}\right] .
\end{aligned}
$$

While deriving (4), the induced magnetic field was linked to the electric field through Faraday's law. It's effect has been self-consistently taken into account in (4) (it appears in the term within square brackets) and the later analysis. This effect is important for the results described in this paper. From here onwards, we assume that the medium is isotropic. Since the electric field only has a $y$ component in the vacuum, $\boldsymbol{J}$ and $\boldsymbol{E}$ will also have only $y$ components in the plasma. The only significant component of (4) is, therefore, $\sigma_{y y}$. Assuming that electrons have a Maxwellian distribution with a temperature $T_{e}$, we find

$$
\sigma_{y y}=\frac{\mathrm{i} q_{e}^{2} n_{e}}{m_{e}(\omega+\mathrm{i} v)}\left[1-W\left(\frac{\omega+\mathrm{i} \nu}{\left|k_{z}\right|\left(k_{B} T_{e} / m_{e}\right)^{1 / 2}}\right)\right]
$$

where $W(z)$ is defined as

$$
W(z)=\frac{1}{\sqrt{2 \pi}} \int_{-\infty}^{\infty} \mathrm{d} x \frac{x}{x-z} \mathrm{e}^{-x^{2} / 2} .
$$

Since the plasma is assumed to be isotropic, we replaced $k_{z}$ with $\left|k_{z}\right|$ in (5). The function $W(z)$ can be computed in terms of the complex error function [9] or the plasma dispersion function [10].

We now return to the 1D scenario. Combining Maxwell's equations, making use of perfectly reflecting boundary conditions [11] and taking the Fourier transform of the resulting expression, we find

$$
E_{y}\left(k_{z}, \omega\right)=\frac{2 \mathrm{i} \omega B_{x}\left(0^{-}\right)}{k_{z}^{2}-\mathrm{i} \omega \mu_{0} \sigma_{y y}}
$$

where $B_{x}\left(0^{-}\right)$is the value of $B_{x}$ at $z=0$ in the vacuum. Taking the inverse Fourier transform of (7), we obtain

$$
E_{y}(z, \omega)=\frac{\mathrm{i} \omega B_{x}\left(0^{-}\right)}{\pi} \int_{-\infty}^{\infty} \mathrm{d} k_{z} \frac{\mathrm{e}^{\mathrm{i} k_{z} z}}{k_{z}^{2}-\mathrm{i} \omega \mu_{0} \sigma_{y y}\left(\left|k_{z}\right|, \omega\right)} .
$$

To solve this integral, we analytically extend the integrand to the lower and upper half in the complex $k_{z}$ plane, and close the loop at infinity in the upper half plane. The solution is the sum of residues at the poles in the upper half plane. The poles are the zeros of

$$
k_{z}^{2}-\mathrm{i} \omega \mu_{0} \sigma_{y y}\left(\left|k_{z}\right|, \omega\right)=0 .
$$

It can be verified that (9) has only one root in the upper half plane. Labelling it $k_{z 1}$, we find that

$$
E_{y}(z, \omega)=-2 \omega B_{x}\left(0^{-}\right) \mathrm{e}^{\mathrm{i} k_{z 1} z}\left|\frac{k_{z}-k_{z 1}}{k_{z}^{2}-\mathrm{i} \omega \mu_{0} \sigma_{y y}}\right|_{k_{z}=k_{z 1}} .
$$

The effective warm-plasma conductivity is given by (5), where $k_{z}$ is the solution of (9) with $\operatorname{Im}\left(k_{z}\right)>0$. Note that all quantities have been transformed back from Fourier space to real space.

The model yields the correct limits for the cold- or collisionless-plasma approximations. In the cold-plasma case, $T_{e} \rightarrow 0$, and the argument of function $W(z)$ in (5) becomes very large. Using (7.1.23) of Abramowitz and Stegun [9], we find that

$$
\lim _{|z| \rightarrow \infty} W(z) \approx-\frac{1}{z^{2}}-\frac{3}{z^{4}}+\ldots
$$




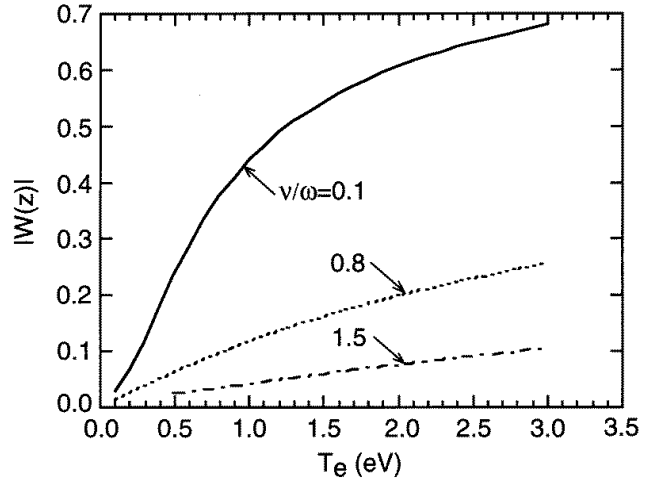

Figure 1. Magnitude of $W(z)$ as a function of $T_{e}$ for $\omega / 2 \pi=13.56 \mathrm{MHz}$ and $n_{e}=3.0 \times 10^{11} \mathrm{~cm}^{-3}$, where $z$ is defined in (5). $|W(z)|$ is the ratio of the warm-plasma conductivity correction ( $\left.\sigma_{\text {warm }}-\sigma_{\text {cold }}\right)$ to the cold-plasma conductivity $\left(\sigma_{\text {cold }}\right)$. If $|W(z)|$ is small, the cold-plasma expression for conductivity is valid. Large values imply that warm-electron effects are important.

For small $T_{e}, W(z)$ scales as $T_{e}$ and $\sigma_{y y}$ is given by

$$
\lim _{T_{e} \rightarrow 0} \sigma_{y y}=\frac{\mathrm{i} q_{e}^{2} n_{e}}{m_{e}(\omega+\mathrm{i} \nu)} .
$$

This is indeed the cold-plasma expression for conductivity.

In the case of a collisionless plasma, $v=0$. If $\left|\omega / k_{z}\right| \ll\left(k_{B} T_{e} / m_{e}\right)^{1 / 2}$, we can use [11]

$$
W(z) \approx \mathrm{i}\left(\frac{\pi}{2}\right)^{1 / 2} z \mathrm{e}^{-z^{2} / 2}+1-z^{2}+\cdots \quad|z|<1
$$

and (9) to obtain

$$
\frac{1}{\delta} \equiv\left|k_{z}\right|=\left[\frac{\omega \omega_{p e}^{2}}{c^{2}}\left(\frac{m_{e} \pi}{2 k_{B} T_{e}}\right)^{1 / 2}\right]^{1 / 3} .
$$

This is the collisionless skin depth as described by Ichimaru [11].

\section{Resonant non-collisional electron heating}

We now connect the model described in the previous section to non-collisional heating. We will concentrate here on the behaviour of the electrons. The physical mechanism of non-collisional heating as represented by this model can best be understood in terms of the electron velocity $v_{y e}$. Using (3), (5) and (6), we find that

$$
\begin{aligned}
v_{y e}= & \frac{\sigma_{y y}}{q_{e} n_{e}} E_{y}=\left[\frac{\mathrm{i} q_{e}}{m_{e}(\omega+\mathrm{i} \nu)}-\frac{\mathrm{i} q_{e}}{\sqrt{2 \pi} m_{e}(\omega+\mathrm{i} \nu)}\right. \\
& \left.\times \int_{-\infty}^{\infty} \mathrm{d}\left(\frac{v_{z}}{v_{t h}}\right) \frac{v_{z}}{v_{z}-(\omega+\mathrm{i} \nu) /\left|k_{z 1}\right|} \mathrm{e}^{-1 / 2\left(v_{z} / v_{t h}\right)^{2}}\right] E_{y}
\end{aligned}
$$

where $v_{t h}=\left(k_{B} T_{e} / m_{e}\right)^{1 / 2}$. Note that $k_{z 1}$ is the solution of (9), and (15) holds in real space. To understand (15), we approach it in stages. We first consider a cold collisionless $\left(T_{e}=0, v=0\right)$ plasma. In this situation, $v_{y e}=$ $\left(\mathrm{i} q_{e} / m_{e} \omega\right) E_{y}$. The electrons, therefore, synchronously oscillate with the electric field in steady state. $v_{y e}$ and
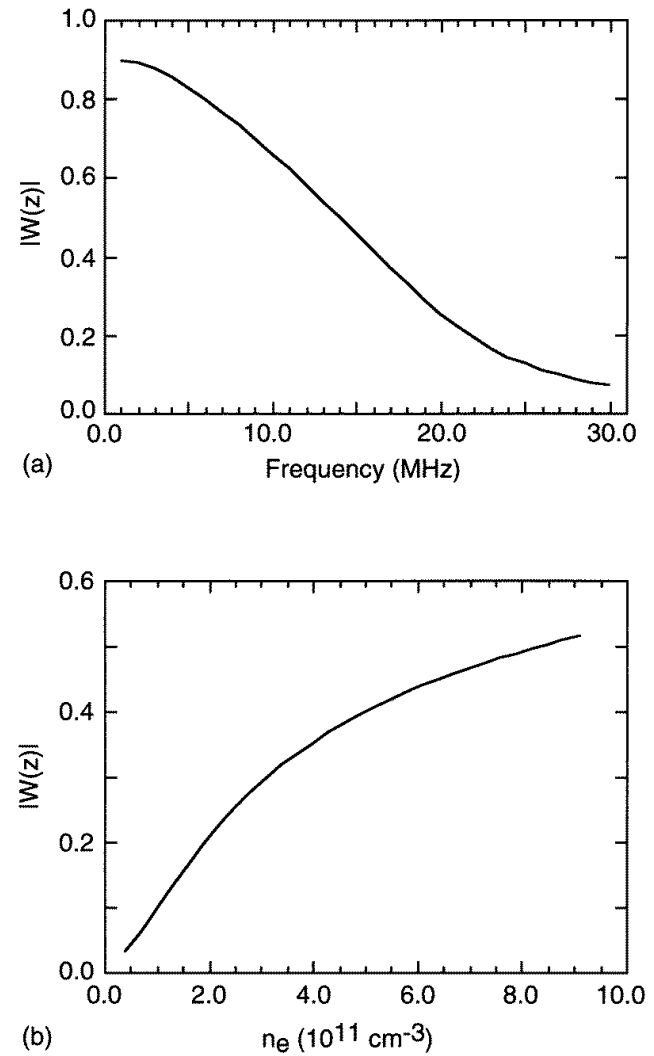

Figure 2. Magnitude of $W(z)$ for $v / \omega=0.3$ and $T_{e}=1.0 \mathrm{eV}$, where $z$ is defined in (5). (a) $|W(z)|$ as a function of frequency for $n_{e}=3.0 \times 10^{11} \mathrm{~cm}^{-3}$. (b) $|W(z)|$ as a function of $n_{e}$ for $\omega / 2 \pi=13.56 \mathrm{MHz}$. Warm-electron effects become less pronounced with increasing effective wave speed, obtained by increasing $\omega$ or decreasing $n_{e}$.

$E_{y}$ are, however, $90^{\circ}$ out of phase. The phase difference is such that the electrons gain energy from the electromagnetic wave in one half of the rf cycle and transfer it back in the other half. The steady state power deposition,

$$
P=\operatorname{Re}\left(\sigma_{y y}\right)\left|E_{y}\right|^{2}
$$

is, therefore, zero. As we add collisions $(v \neq 0)$, electrons start experiencing a viscous drag which has two consequences. First, in order to maintain a steady-state oscillation, a constant power input is required $(P \neq 0)$. Second, collisional drag leads to a phase alignment of the electron velocity and electric field.

In a warm collisionless $\left(T_{e} \neq 0, n \neq 0\right)$ plasma, the second term in (15) also contributes to electron motion. This term is an ensemble average of the contribution from all electrons at that location. The largest contribution comes from electrons whose velocities are comparable to $\omega /\left|k_{z 1}\right|$, which we will refer to as the 'effective' phase speed. These electrons interact resonantly with the wave, as is evident from the $\left(v_{z}-\omega /\left|k_{z 1}\right|\right)$ term in the denominator. To learn what is special about these electrons, we consider two extreme situations. If $k_{z 1 \text { (real) }} \gg k_{z 1(\mathrm{imag})}$, $\omega /\left|k_{z 1}\right|$ is the phase speed of a travelling electromagnetic wave. Electrons whose velocities are comparable to 


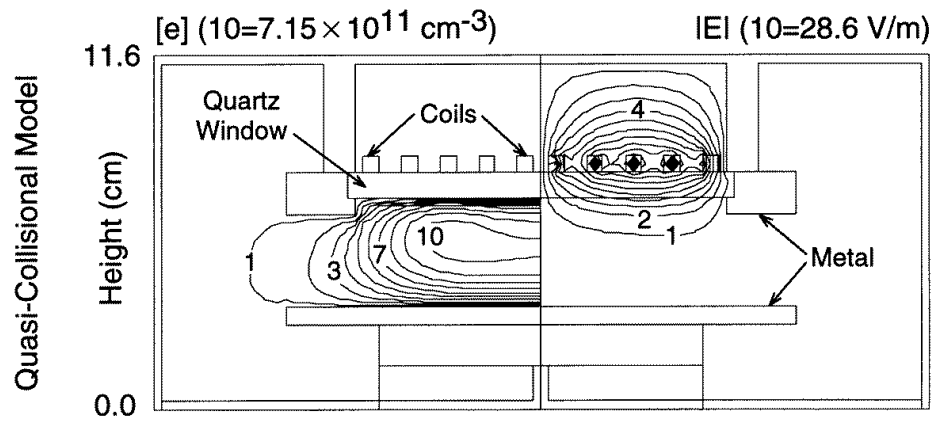

(a)

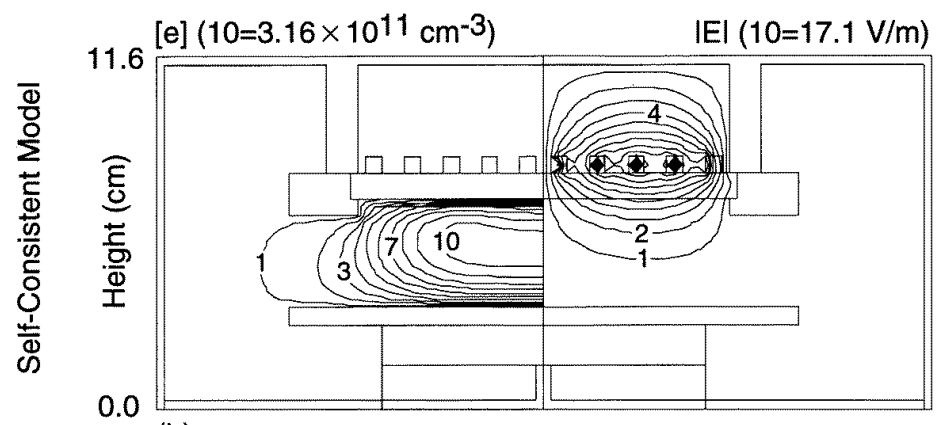

(b)

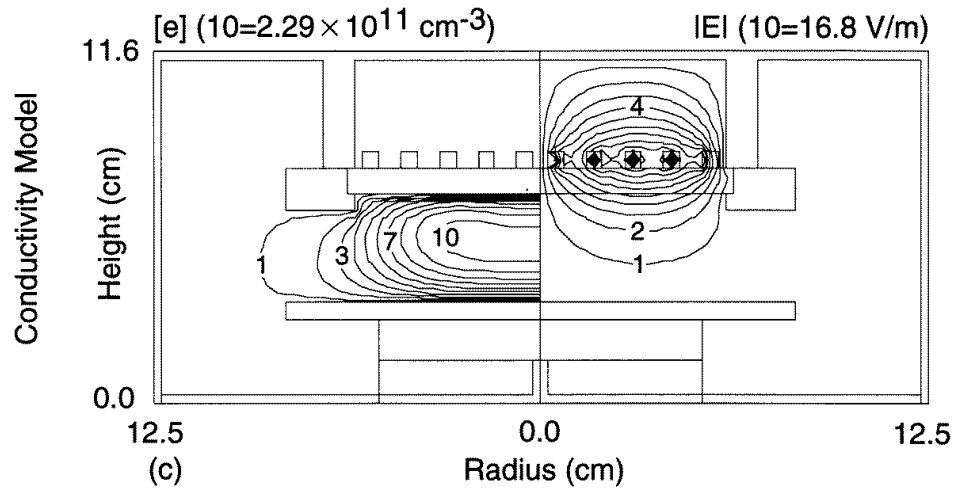

Figure 3. Electron density and electric field amplitude computed using the (a) quasi-collisional, (b) self-consistent and (c) conductivity models. The pressure is 5 mTorr, the inductively coupled power is $300 \mathrm{~W}$ and the simulation has been done in Ar.

$\omega /\left|k_{z 1}\right|$ experience essentially dc electric fields, and gain net directed energy from them. If $k_{z 1(\text { imag })} \gg k_{z 1(\text { real })}$, the electromagnetic wave decays exponentially in the $z$ direction. Electrons whose velocities are comparable to $\omega /\left|k_{z 1}\right|$ constitute that group which can gain directed energy from the electromagnetic wave during one half of the rf cycle and leave the region where electron acceleration occurs before the electric field reverses its direction. For a general $k_{z 1}$, the situation will be more complicated. The resonance will, however, be present. Non-collisional heating can, therefore, be interpreted as a consequence of a resonant interaction of a subset of warm electrons with the electromagnetic wave. As in a cold plasma, collisions introduce drag and lead to the conversion of this directed electron energy to random thermal energy. Note that no structure is assumed for the electric field in (15). Non-collisional heating can take place in this configuration even when the electric field is homogeneous $\left(k_{z 1(\text { real })} \gg k_{z 1(\mathrm{imag})}\right)$.
We now use the model to investigate the effect of electron temperature, electron density, collision frequency and frequency on non-collisional heating. We will do so in terms of the magnitude of $W(z)$ in (5). $W(z)$ is the ratio of the warm-plasma conductivity correction $\left(\sigma_{\text {warm }}-\sigma_{\text {cold }}\right)$ to the cold-plasma conductivity $\left(\sigma_{\text {cold }}\right)$, where $\sigma_{\text {cold }}$ is defined in (12). If $|W(z)|$ is small, $\sigma_{\text {warm }} \approx \sigma_{\text {cold }}$, and the cold-plasma expression for conductivity is valid. Large values of $|W(z)|$ imply that warm-plasma effects are important. The magnitude of $W(z)$ as a function of electron temperature is shown in figure 1 for several values of $v / \omega$. For all values of $v / \omega,|W(z)|$ increases when $T_{e}$ is increased. In our representation, non-collisional heating relies on the fact that warm electrons gain net directed energy from the electromagnetic fields through a resonant interaction. As the electron temperature increases, the proportion of electrons with speeds comparable to the effective wave phase speed increases. This makes the non-collisional heating process more efficient and $|W(z)|$ 
increases. The same effect occurs if the effective wave phase speed decreases. The effective wave phase speed $\left(\omega /\left|k_{z}\right|\right)$ can decrease due to either a decrease in frequency or an increase in $\left|k_{z}\right|$. The effect of frequency is shown in figure 2(a) where $|W(z)|$ increases with decreasing frequency. According to (9), one factor that can increase $\left|k_{z}\right|$ is an increase in electron density. This is the reason $|W(z)|$ increases with increasing $n_{e}$ as shown in figure 2(b).

Maintaining a phase correlation between the electrons and electromagnetic wave is essential for the resonant interaction to take place. Collisions dislodge resonantly interacting electrons from their position in phase space and make the non-collisional process less efficient. We, therefore, find in figure 1 that, for a given $T_{e},|W(z)|$ decreases as the collision frequency is increased.

\section{Application to ICP reactor modelling}

Low-pressure inductively coupled plasmas are widely used in the microelectronics fabrication industry. To understand the operation of these plasma reactors and improve their performance, considerable effort has been put into the numerical modelling of these devices [12-14]. An important issue in these models is how the plasma dynamics are coupled with the electromagnetic fields so that kinetic information about non-collisional heating is properly communicated to Maxwell equations,

$$
\nabla^{2} \boldsymbol{E}+\left(\omega^{2} / c^{2}\right) \boldsymbol{E}=-\mathrm{i} \omega \mu_{0} \boldsymbol{J}
$$

This problem was first addressed by Vahedi et al [3]. They took account of non-collisional heating effects through an effective collision frequency in the cold-plasma expression for conductivity, (12). The effective collision frequency was derived using a 1D phenomenological model and a specific profile was assumed for the electric field. Rauf and Kushner [4] used a Monte Carlo simulation to kinetically compute plasma current. This plasma current was used directly in (17) to determine the electric fields. The analytical model in this paper suggests an alternative method for coupling a kinetic plasma simulation with the electromagnetic field computation: one can treat (5) as the general expression for warm-plasma conductivity and use it to couple $\boldsymbol{J}$ with $\boldsymbol{E}$ in (17). The resulting equation can then be solved for $\boldsymbol{E}$. A number of approximations are involved here. First, (5) is strictly valid only for the 1D situation considered in this paper. Also, (5) has been derived for a homogeneous plasma and Maxwellian electron energy distribution. The results described below, however, show that the resulting computational model is reasonably accurate. Because of these approximations, the use of this approach is only warranted if electron current density cannot be computed kinetically. Otherwise, the technique described in [4] will prove to be more effective.

To test the approach described above, we used (5) to couple an electron Monte Carlo simulation (EMCS) with Maxwell equations in the hybrid plasma equipment model (HPEM) [12], a comprehensive plasma simulation tool. Simulations were performed for an inductively coupled Gaseous Electronics Conference reference cell with a fiveturn antenna. The reactor is described in more detail by
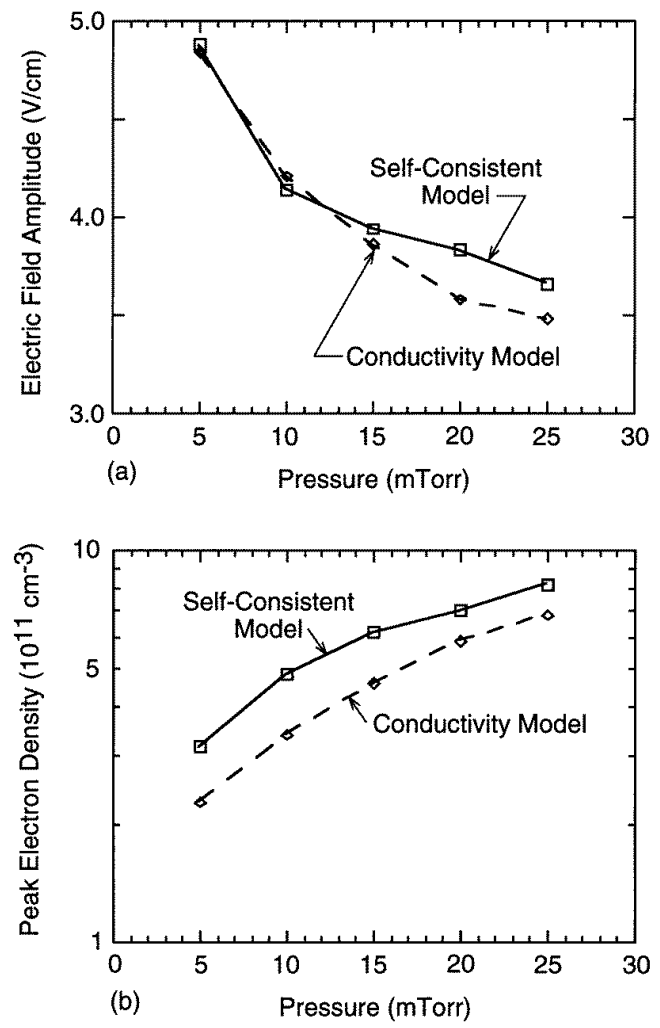

Figure 4. Electric field amplitude at $(r=3 \mathrm{~cm}, z=6.47 \mathrm{~cm})$ and peak plasma density as a function of pressure from the conductivity and self-consistent models. These results are from simulations of argon in the inductively coupled GEC reference cell. The inductively coupled power is $300 \mathrm{~W}$.

Miller et al [15]. The electric field amplitude and electron density profile at 5 mTorr in Ar are shown in figure 3 using three different models. Inductively coupled power, defined as $\int \boldsymbol{J} \cdot \boldsymbol{E} \mathrm{d} r^{3}$, is kept constant at $300 \mathrm{~W}$. The three models only differ in the manner in which the EMCS is coupled to Maxwell equations. In the 'conductivity model', the warm-plasma conductivity, (5), is used for coupling. The 'self-consistent model' [4] makes use of a kinetically computed electron current density for coupling. Since this model does not make any assumptions about the nature of the interaction, its predictions can be assumed to be the most accurate of the three. In the 'quasi-collisional model', we use the cold-plasma conductivity, (12), to couple the EMCS and Maxwell's equations. This model does not communicate non-collisional heating effects to the Maxwell equations. We find that both the self-consistent and conductivity models yield nearly the same electric field and plasma density, both of which are significantly smaller than the quasi-collisional model. The large difference in densities is a consequence of the fact that inductive power deposition, which is kept constant here, is defined as $\int \boldsymbol{J} \cdot \boldsymbol{E} \mathrm{d} r^{3}$, where $\boldsymbol{J}=\sigma_{\text {cold }} \boldsymbol{E}$ in the quasi-collisional model. This power only takes account of collisional heating in the quasi-collisional model. Since the kinetic electron Monte Carlo simulation includes all heating effects and non-collisional heating is prominent at $5 \mathrm{mTorr}$ in $\mathrm{Ar}$, 
significantly more power (almost three times) is deposited into the electrons [4]. This leads to the large density observed for the quasi-collisional model in figure 3(a).

The peak electron density and the electric field amplitude at a point in the plasma $0.4 \mathrm{~cm}$ below the quartz window, in line with the centre coil $(r=3 \mathrm{~cm}, z=$ $6.47 \mathrm{~cm}$ ), are plotted in figure 4 as a function of pressure for the conductivity and self-consistent models. The results of the quasi-collisional model under similar conditions are shown in [4]. The trends for the conductivity and selfconsistent models are similar. The actual magnitudes are, however, slightly different, particularly in the peak electron density. This is mainly due to the approximations that are described above. Under similar conditions, the model of Vahedi et al [3] compares favourably with the results of the conductivity model [16].

\section{Conclusions}

We described a self-consistent analytical model for noncollisional heating in plasmas. The model was used to investigate the mechanism of non-collisional heating and to determine the regimes in which non-collisional heating effects are dominant. It was found that non-collisional heating can be described as a consequence of a resonant interaction of electrons with the electromagnetic wave. The electromagnetic wave imparts directed energy to the electrons which have velocities comparable to the effective wave phase speed. This directed energy is randomized by collisions, leading to electron heating. It was also found that electron heating is efficient if the proportion of electrons participating in the resonant interaction process is large, and there are not many collisions that destroy the essential phase correlation between the wave and electrons. On a practical note, the analytical model yielded an expression for warm-plasma conductivity. It was shown that this expression can be used in plasma simulations to take account of non-collisional heating effects.

\section{Acknowledgments}

This work was supported by ARPA/AFOSR (F9620-95-10524), the National Institute of Standards and Technology, the National Science Foundation (ECS 94-04133) and the Semiconductor Research Corporation.

\section{References}

[1] Turner M M 1993 Phys. Rev. Lett. 711844

[2] Godyak V A, Piejak R B and Alexandrovich B M 1994 Plasma Sources Sci. Technol. 3169

[3] Vahedi V, Lieberman M A, DiPeso G, Rognlien T D and Hewett D 1995 J. Appl. Phys. 781446

[4] Rauf S and Kushner M J 1997 J. Appl. Phys. 815966

[5] Shaing K C 1996 Phys. Plasmas 33300

[6] Kolobov V I, Lymeropoulos D P and Economou D J 1997 Phys. Rev. E 553408

[7] Kolobov V I and Economou D J 1997 Plasma Sources Sci. Technol. $6 \mathrm{R} 1$

[8] Weibel E S 1967 Phys. Fluids 10741

[9] Abramowitz M and Stegun I A 1964 Handbook of Mathematical Functions with Formulas, Graphs and Mathematical Tables (Washington, DC: National Bureau of Standards)

[10] Fried B D and Conte S D 1961 The Plasma Dispersion Function (New York: Academic)

[11] Ichimaru S 1973 Basic Principles of Plasma Physics: a Statistical Approach (Reading, MA: Academic)

[12] Kushner M J, Collison W Z, Grapperhaus M J, Holland J P and Barnes M S 1996 J. Appl. Phys. 801337

[13] Stewart R A, Vitello P, Graves D B, Jaeger E F and Berry L A 1995 Plasma Sources Sci. Technol. 436

[14] Lymberopoulos D P and Economou D J 1994 J. Vac. Sci. Technol. A 121229

[15] Miller P A, Hebner G A, Greenberg K E, Pochan P D and Aragon B A 1995 J. Res. NIST 100427

[16] Rauf S and Kushner M J 1996 Bull. Am. Phys. Soc. 411325 